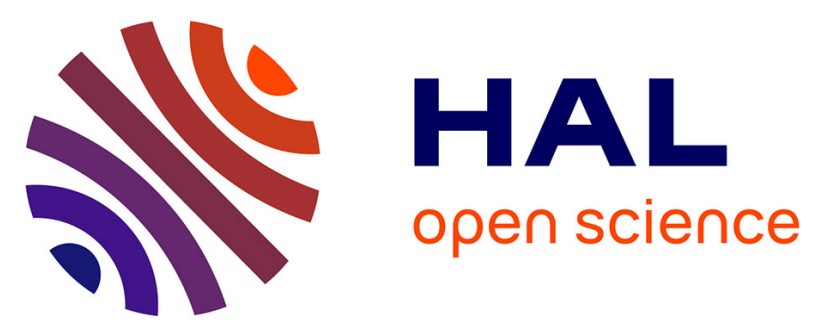

\title{
Stochastic Current-Induced Magnetization Switching in a Single Semiconducting Ferromagnetic Layer
}

J. Gorchon, J. Curiale, A. Lemaître, N. Moisan, M. Cubukcu, G. Malinowski, C. Ulysse, G. Faini, H. j. von Bardeleben, V. Jeudy

\section{- To cite this version:}

J. Gorchon, J. Curiale, A. Lemaître, N. Moisan, M. Cubukcu, et al.. Stochastic Current-Induced Magnetization Switching in a Single Semiconducting Ferromagnetic Layer. Physical Review Letters, 2014, 112 (2), pp.026601 10.1103/PhysRevLett.112.026601 . hal-01381247

\section{HAL Id: hal-01381247 \\ https://hal.univ-lorraine.fr/hal-01381247}

Submitted on 14 Oct 2016

HAL is a multi-disciplinary open access archive for the deposit and dissemination of scientific research documents, whether they are published or not. The documents may come from teaching and research institutions in France or abroad, or from public or private research centers.
L'archive ouverte pluridisciplinaire HAL, est destinée au dépôt et à la diffusion de documents scientifiques de niveau recherche, publiés ou non, émanant des établissements d'enseignement et de recherche français ou étrangers, des laboratoires publics ou privés. 


\title{
Stochastic Current-Induced Magnetization Switching in a Single Semiconducting Ferromagnetic Layer
}

\author{
J. Gorchon, ${ }^{1}$ J. Curiale,${ }^{2,1,3}$ A. Lemaitre, ${ }^{2}$ N. Moisan, ${ }^{1}$ M. Cubukcu, ${ }^{4}$ G. Malinowski, ${ }^{1}$ C. Ulysse, ${ }^{2}$ G. Faini, ${ }^{2}$ \\ H. J. von Bardeleben, ${ }^{4}$ and V. Jeudy ${ }^{1,5, *}$ \\ ${ }^{1}$ Laboratoire de Physique des Solides, Université Paris-Sud, CNRS, UMR8502, 91405 Orsay, France \\ ${ }^{2}$ Laboratoire de Photonique et de Nanostructures, CNRS, UPR 20, 91460 Marcoussis, France \\ ${ }^{3}$ Consejo Nacional de Investigaciones Científicas y Técnicas, Centro Atómico Bariloche-Comisíon Nacional de Energía Atómica, \\ Avenida Bustillo 9500, 8400 San Carlos de Bariloche, Río Negro, Argentina \\ ${ }^{4}$ Institut des nanosciences de Paris, Université Pierre et Marie Curie, CNRS, UMR7588, 75252 Paris, France \\ ${ }^{5}$ Université Cergy-Pontoise, 95000 Cergy-Pontoise, France \\ (Received 28 August 2013; published 14 January 2014)
}

\begin{abstract}
We show experimental evidence of magnetization switching in a single $(\mathrm{Ga}, \mathrm{Mn})(\mathrm{As}, \mathrm{P})$ semiconducting ferromagnetic layer, attributed to a strong reduction of the magnetization and the anisotropy due to current injection. The nucleation of magnetization reversal is found to occur even in the absence of a magnetic field and to be both anisotropic and stochastic. Our findings highlight a new mechanism of magnetization manipulation based on spin accumulation in a semiconductor material.
\end{abstract}

DOI: 10.1103/PhysRevLett.112.026601

In spin electronics, a large variety of stimuli has been demonstrated to be efficient in manipulating a magnetic state, such as spin polarized currents [1-3], ultrafast laser pulses [4], and electric fields [5,6], thereby opening challenging questions on the physical processes involved in magnetization excitations [7-9] and reversal [2,10-17]. In particular, current-induced magnetization reversals in nanopillars or domain-wall motion in tracks originate from a spin transfer torque (STT) $[18,19]$ exerted on local magnetic moments by accumulated out-of-equilibrium carrier spins. STT effects [20-22] have been explored in metallic $[10,13,16]$ and in semi-conducting $[2,14,15]$ magnetic multilayer nanopillar structures. While most of these experiments aim at understanding current-induced magnetization reversal in a deterministic way, reports on stochastic reversal remain scarce $[11,12,17]$. However, the study of stochastic reversal $[11,12]$ is particularly fruitful since it gives access to the potential barrier to spontaneous switching.

In order to observe stochastic magnetization reversal in a ferromagnetic semiconductor layer, we fabricated the device presented in Figs. 1(a)-(b). It consists of two regions, a nucleation area located beneath a narrow (2 $\mu$ m-wide) $\mathrm{Au} / \mathrm{Ti}$ electrode, and a semicircular $(\mathrm{Ga}$, $\mathrm{Mn})(\mathrm{As}, \mathrm{P})$ area for domain expansion and visualization. The $\left(\mathrm{Ga}_{0,9}, \mathrm{Mn}_{0,1}\right)\left(\mathrm{As}_{0,9}, \mathrm{P}_{0,1}\right)$ film is $50 \mathrm{~nm}$ thick and was grown by low-temperature $\left(T=250^{\circ} \mathrm{C}\right)$ molecular beam epitaxy on top of a $\mathrm{GaAs}(001)$ substrate [23]. The film was then annealed at $T=250{ }^{\circ} \mathrm{C}$, during $1 \mathrm{~h}$ and presents a perpendicular magnetic anisotropy. Its Curie temperature $T_{c}$ is $119 \pm 1 \mathrm{~K}$. The semicircular geometry was patterned by electron beam lithography. The electrical connections consist in a $10 \mathrm{~nm}$ thick titanium film covered by a $250 \mathrm{~nm}$
PACS numbers: 72.25.Dc, 75.50.Pp, 75.60.Ch, 75.78.Fg

thick gold layer. For details on experimental methods, see Supplemental Material Ref. [24].

Evidence for current induced stochastic magnetization reversal is reported in Figs. 1(c)-1(d). Figure 1(c) shows the sample magnetic state after injection of a current pulse with three different durations in a homogeneously magnetized layer. In these experiments and the following the current was always injected from the semicircular electrode to the narrow one. Clearly resolved semi-ring-shaped domains centered around the narrow electrode can be observed. Their number and spatial extension increase with pulse duration. Obviously, the generation of these domains arises from the repetition of a nucleation and/or propagation event induced by the current: during the current pulse, a domain with opposite magnetization nucleates underneath the narrow electrode, then its domain wall (DW) is pushed away from the electrode by STT, leaving a large homogeneously magnetized region before the next nucleation event. Let us note that the DW displacement varies nonlinearly with the pulse duration, a consequence of the semicircular geometry: indeed, as the current density decays as the inverse of the distance from the narrow electrode, the STT hence the DW velocity decreases accordingly. Close to the narrow electrode, for typical current pulse amplitudes, $\left(\approx 2 \mathrm{GA} / \mathrm{m}^{2}\right)$, DWs almost follow a flow regime, with velocities of the order [25] of $1 \mathrm{~m} / \mathrm{s}$. In the semicircular area, the DWs move in creep regimes.

In order to perform a statistical analysis of magnetization reversal, the time evolution under dc bias current of magnetization direction was recorded close to the narrow electrode [as schematized in Figs. 1(c) and (d)]. The nucleation event is stochastic as revealed in Fig. 1(d). The Kerr intensity exhibits a random telegraph noise. This 
(a)

(b)

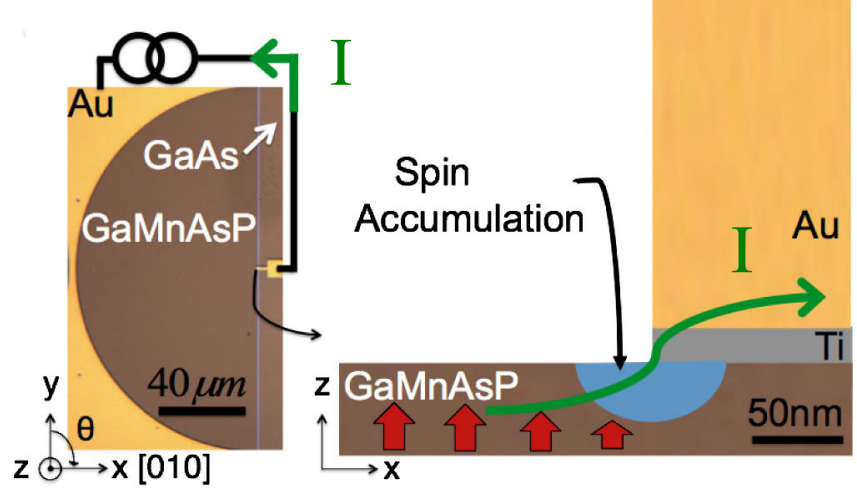

(c)

(d)
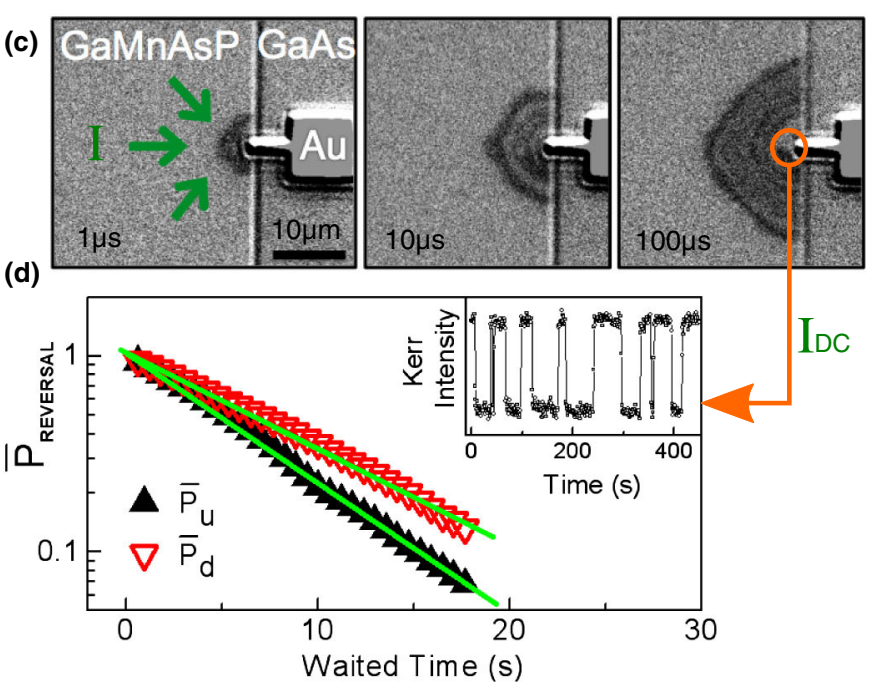

FIG. 1 (color online). Experimental set-up and stochastic magnetization reversal. (a) Optical micrograph of the (Ga,Mn)(As,P) layer and schematic of the electrical connections showing the semicircular (left) and narrow (right) Au/Ti electrodes. (b) Cross section of the narrow electrode featuring the current flow, the volume $\left(V_{\mathrm{SF}} \approx w l_{\mathrm{SF}}^{2}\right.$, see text) where hole spins accumulate (semicircle not to scale) and the decrease of the magnetization (arrows). (c) Magnetic domains observed by polar magneto-optical Kerr microscopy. The two gray levels (up/down) correspond to magnetization pointing towards the $+z$ and $-z$ directions. The sample is initially in a saturated magnetic state (homogeneous bright gray level). The injected current pulses with an intensity of $I=2.164 \mathrm{~mA}$ and durations of $1 \mu \mathrm{s}, 10 \mu \mathrm{s}$ and $100 \mu \mathrm{s}$ produce a set of quasi-circular domains revealing successive magnetization switching. Images were acquired $30 \mathrm{~s}$ after the current pulses were injected. Initial sample temperature $T_{0}=95 \mathrm{~K}$. No magnetic field is applied. (d) Inset. Typical random telegraph noise reflecting variations of the gray level in a $100 \mu \mathrm{m}^{2}$ square close to the interface. Semi-log plot of the typical probability of nonreversal $\left(\bar{P}_{\text {up }}\right.$ and $\left.\bar{P}_{\text {down }}\right)$ as a function of the waited time $(t)$ since the last reversal. Each curve is deduced from $\approx 50$ switches. The (green) lines are exponential fits.

noise reflects the stochastic switching of the magnetization between the film easy directions (up/down), perpendicular to the film. For each of those two magnetic states, the probabilities of non-reversal from the up $(u)$ and down $(d)$ states $\left(\bar{P}_{u}\right.$ and $\left.\bar{P}_{d}\right)$ were recorded as a function of the time $(t)$ between two subsequent reversals. As reported in Fig. 1(d), $\bar{P}_{u}$ and $\bar{P}_{d}$ decrease exponentially with $t$. The good agreement with a $\bar{P}_{u, d}=1-e^{-t / \tau_{u, d}}$ law, strongly suggests that the random telegraph noise can be described by the Néel-Brown model with a unique dwell time given by:

$$
\tau_{u, d}=\tau_{0} e^{\frac{\Delta E_{u, d}}{k_{B} \cdot T}}
$$

where $k_{B}$ is the Boltzmann constant and $\Delta E_{u, d}$ the energy barrier heights characterizing each state. Within this framework, the experimental determination of the dwell times $\tau_{u, d}$ gives access to the variations of $\Delta E_{u, d}$ which depends on the external parameters such as magnetic fields perpendicular $\left(\mu_{0} H_{z}\right)$ and parallel $\left(\mu_{0} H_{x y}\right)$ to the film, the bias current $(I)$ and the temperature $(T)$, as reported in Fig. 2.

Here, it is important to stress that the temperature rise $\left(\Delta T_{i}\right)$ due to Joule heating, at the interface between the narrow electrode and the magnetic layer, was systematically taken into account. As detailed in Ref. [24], the values of $\Delta T_{i}$ were deduced from a comparison between electrical measurements and finite element simulations (see Ref. [26] for details). In the explored bias current range (0.9-2.1 mA) and cryostat temperature range $\left(T_{0}=93.5-78 \mathrm{~K}\right)$, the interface temperature $\left(T=T_{0}+\Delta T_{i}\right)$ extends from 96 to $89 \mathrm{~K}$. As those values remain well below $T_{c}$, a magnetic switching produced by a spatially localized paramagnetic phase is excluded.

A perpendicular magnetic field $\left(\mu_{0} H_{z}\right)$ produces asymmetric and linear variations of $\ln \left(\tau_{u, d}\right)$ for the up and down states [see Fig. 2(a)]. The asymmetry indicates that a magnetic state is more stable for $\mu_{0} H_{z}$ pointing in the direction of the magnetization. The linear variations reflect the proportionality between the energy barrier height $\Delta E_{u, d}$ and $\mu_{0} H_{z}$, as expected for a Zeeman-like contribution. On the other hand, the curves $\tau_{u}$ and $\tau_{d}$ do not cross at $\mu_{0} H_{z}=0 \mathrm{mT}$. Typical offset field $\left(H_{0 z}\right)$ magnitudes were found to be of the order of $0.15-0.3 \mathrm{mT}$ for the investigated bias current range (0.9-2.1 mA). As shown in Ref. [24], $H_{0 z}$ corresponds to the out-of-plane component of the Oersted field.

The evolution of the dwell time with bias current $(I)$ is reported in Fig. 2(b) for different temperatures. The bias current produces identical variations of $\ln \left(\tau_{u, d}\right)$ for both up and down magnetization directions. The linear decrease of $\ln \left(\tau_{u, d}\right)$ with increasing current reflects the reduction of the barrier heights and will be addressed later.

The contribution of an in-plane applied magnetic field $\left(\mu_{0} H_{x y}\right)$ to magnetization reversal differs from that of the other external parameters, as observed in Fig. 2(c). 

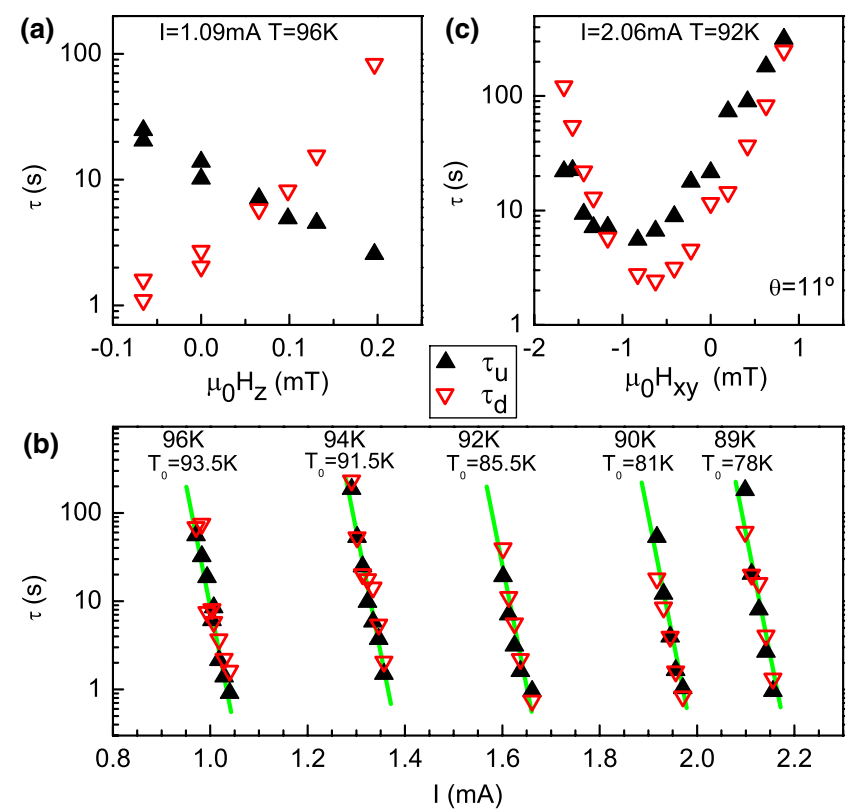

FIG. 2 (color online). Variation of the dwell times with the external parameters (in semilogarithmic scales). (a) Magnetic field perpendicular to the film $\mu_{0} H_{z}$. (b) Bias current $I$. Interface temperatures and cryostat temperatures $\left(T_{0}\right)$ are indicated on top of the curves. The solid lines are fits of Eq. (3). (c) Magnetic field parallel to the film $\mu_{0} H_{x y} . \theta$ is the angle between $\mu_{0} H_{x y}$ and the [100] crystallographic direction.

The curves are not symmetric around $\mu_{0} H_{x y}=0 \mathrm{mT}$. Their minimum corresponds to an offset field $\left(\mu_{0} H_{0 x y}\right)$ associated to the Oersted field, as already discussed [24]. Surprisingly, $\ln \left(\tau_{u}\right)$ and $\ln \left(\tau_{d}\right)$ exhibit a strong nonlinear variation with $\mu_{0} H_{x y}$ and are split. In order to address separately both contributions, it is convenient to define a reduced dwell time $\ln \left(\tau_{\text {diff }}\right)=\left(\Delta E_{u}-\Delta E_{d}\right) / k_{B} T$, associated to the asymmetry of the energy barrier heights and a dwell time $\ln \left(\tau_{\text {mean }}\right)=\ln \left(\tau_{0}\right)+\left(\Delta E_{u}+\Delta E_{d}\right) / 2 k_{B} T$, associated to the mean energy barrier.

As shown in Fig. 3(a), $\ln \left(\tau_{\text {diff }}\right)$ presents a linear variation with $\mu_{0} H_{x y}$. This reflects an asymmetry between the energy barrier heights $\Delta E_{u}$ and $\Delta E_{d}$ proportional to the magnetic field, as expected for an additional Zeeman-like torque. Interestingly, the slope of $\ln \left(\tau_{\text {diff }}\right)$ is found to vary with the angle between the in-plane field and the [110] in-plane crystallographic direction of the film. This may originate from the in-plane anisotropy of the $(\mathrm{Ga}, \mathrm{Mn})(\mathrm{As}, \mathrm{P})$ monocrystalline film. Indeed, as the explored magnetic field range $\mu_{0} H_{x y}=0-2 \mathrm{mT}$ is close to the second and fourth order in-plane anisotropy field-values $\left(\mu_{0} H_{2 p}=7 \mathrm{mT}\right.$ and $\mu_{0} H_{4 p}=2 \mathrm{mT}$, see Refs. [24,27]), $\mu_{0} H_{x y}$ is expected to significantly modify the energy barrier heights. Therefore the variation of $\ln \left(\tau_{\text {diff }}\right)$ with the direction of the field $\mu_{0} H_{x y}$ strongly suggests the in-plane anisotropy to result in an anisotropic probability of nucleation.

We now discuss the variations of the mean magnetization reversal rate with an in-plane field $\mu_{0} H_{x y}$. As shown in
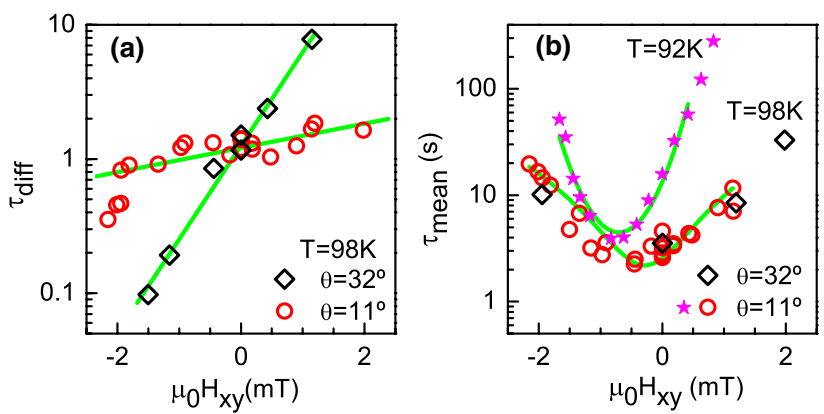

FIG. 3 (color online). In-plane magnetic field contributions to the dwell times. (a) $\tau_{\text {diff }}$, in semilogarithmic scale and (b) $\tau_{\text {mean }}$ (see text). In (a), the solid lines are guides for eyes. In (b), the solid lines are fits based on Eq. (3) for $T=98 \mathrm{~K}, I=0.85 \mathrm{~mA}$ and for $T=92 \mathrm{~K}, I=2.06 \mathrm{~mA}$. The angles between the applied magnetic field and the [110] crystallographic direction are indicated at the bottom right of each graph.

Fig. 3(b), $\ln \left(\tau_{\text {mean }}\right)$ varies nonlinearly with $\mu_{0} H_{x y}$. In order to determine the origin of this variation and more generally the mechanisms involved in stochastic magnetization reversals, it is important to estimate to what extent, the unpolarized spin carriers flowing from the electrode modify the magnetic state of the $(\mathrm{Ga}, \mathrm{Mn})(\mathrm{As}, \mathrm{P})$ ferromagnetic layer. The spatial evolution of the magnetic state with increasing distance from the electrode is schematically depicted in Fig. 4.

In $(\mathrm{Ga}, \mathrm{Mn})(\mathrm{As}, \mathrm{P})$, ferromagnetism originates from the exchange coupling between the manganese magnetic moments and the spin of the carriers belonging to the valence band [27], or from impurity states close to the valence band [28]. After tunneling through the interface, unpolarized electrons produce a spin depolarization in the ferromagnet. This is due to the spin accumulation of out-ofequilibrium carriers spins [29] occurring over the spin diffusion length $\ell_{\mathrm{SF}}(\approx 10 \mathrm{~nm})$ [30]. The associated spin flip time $\left(\tau_{\mathrm{SF}}\right)$ is of the order [31] of $50 \mathrm{fs}$. Conventionally, theoretical descriptions of the STT [18-22] assume the magnetization $\vec{M}_{\text {eq }}$ modulus and the micromagnetic parameters to remain unaffected by the spin accumulation. For a dc



FIG. 4 (color online). Ferromagnet magnetic states close to the interface. In a simplified way, three areas can be distinguished: (1) for $0<l<l_{\mathrm{SF}}$, the spin accumulation produces a strong outof-equilibrium Mn-carrier spins system; (2) for $l_{\mathrm{SF}}<l<l_{\text {relax }}$, the $\mathrm{Mn}$ and carrier spins systems are coupled and the magnetization relaxes towards equilibrium; and (3) for $l>l_{\text {relax }}$, the magnetization is at equilibrium. 
current flow and a stationary accumulation, no STT is expected at a distance from the interface larger than $l_{\mathrm{SF}}$. With our geometry, the current injection over the width $w$ of the narrow electrode should produce spin accumulation over the volume $V_{\mathrm{SF}} \approx w l_{\mathrm{SF}}^{2}$, as depicted in Fig. 1(b).

However, a sufficiently large spin depolarization can also reduce significantly [27] both the magnetization $(M)$ and the magnetic anisotropy $\left(K_{u} \sim M^{2}\right)$. Consequently, the domain wall energy $\left(\sigma \sim \sqrt{K_{u}}\right)$ and the height of the nucleation energy barriers should decrease and stochastic magnetization switching be favored. The associated out-ofequilibrium magnetic states encountered as the distance $l$ from the interface increases are considered in Fig. 4. In the present case of $(\mathrm{Ga}, \mathrm{Mn})(\mathrm{As}, \mathrm{P})$, for $0<l<l_{\mathrm{SF}}$, spins accumulate and create a strong out-of-equilibrium coupled $\mathrm{Mn}$-carrier spins system. The out-of-equilibrium magnetization relaxes over a time scale $\tau_{\text {relax }}(\approx 2 \mathrm{~ns})$ much larger than $\tau_{\mathrm{SF}}$, as deduced from pump-probe experiments [24]. Due to the carrier spin diffusion and drift, the coupled outof-equilibrium state extends over a distance $l=l_{\text {relax }}$ much larger than $l_{\mathrm{SF}}$. For $l>l_{\text {relax }}$, the magnetic state has been fully recovered.

To discriminate between those two possibilities, namely a weakly perturbed or a strongly modified magnetic state, the decrease of the energy barrier produced by the dc current over the volume $V_{\mathrm{SF}}$ has to be compared to the magnetostatic and anisotropy energies of the system, at equilibrium. As shown in Fig. 2(b), the mean heights of energy barriers are decreasing linearly with the current. The magnitude of the decrease is given by $\partial \ln \left(\tau_{\text {mean }}\right) / \partial I=-a / k_{B} T$. For the best fits of $a / k_{B} T\left(=55 \pm 5 \mathrm{~mA}^{-1}\right)$ and for the explored current range (0.9-2.1 mA), the decrease of the barrier height is $\delta E=400-900 \mathrm{meV}$. The corresponding energy decrease per unit volume $\delta E / V_{\mathrm{SF}}$ is of the order of $300-700 \mathrm{~J} / \mathrm{m}^{3}$. These values are close to the magnetostatic energy $\left(=\mu_{0} M^{2} / 2=330 \mathrm{~J} / \mathrm{m}^{3}\right)$ and the perpendicular anisotropy energy $\left(K_{u}=1530 \mathrm{~J} / \mathrm{m}^{3}\right)$, deduced from magnetometry and ferromagnetic resonance measurements (see Ref. [24]). Therefore the spin depolarization results in a strong modification of the magnetic state of the ferromagnet. Note also that an important contribution of magnons can be excluded since the observed variation of $\partial \ln \left(\tau_{\text {mean }}\right) / \partial I$ with the bias current is rather weak $(\approx 10 \%)$. Indeed, following Ref. [11], $I \sim n_{m} \sim T_{m}^{3 / 2}$, where $n_{m}$ and $T_{m}$ are the density and the temperature of magnons, respectively. Replacing $T$ by $T_{m}$ and $\Delta E_{u, d}$ by $\Delta E_{0}-a I$ in Eq. (1) would lead to a variation of $\partial \ln \left(\tau_{\text {mean }}\right) / \partial I$ by a factor larger than 1.7 for the explored current range.

In order to explore a possible contribution of the Hanle effect to the variation of $\ln \left(\tau_{\text {mean }}\right)$ with $\mu_{0} H_{x y}$ [observed in Fig. 3(b)], we propose a simple model, assuming a negligible magnetic anisotropy. For a magnetization relaxation dominated by spin decoherence, the response of the magnetization $\left(\vec{M}=\vec{M}_{\mathrm{eq}}+\overrightarrow{\delta M}\right)$ to an in-plane field $\vec{H}_{x y}$ is given by [24],

$$
\frac{\partial \vec{M}}{\partial t}=\gamma \vec{H}_{x y} \times \vec{M}-\frac{\overrightarrow{\delta M}}{\tau_{\mathrm{dec}}}
$$

In Eq. (2), $\tau_{\text {dec }}$ is the decoherence time, and $\gamma$ is the gyromagnetic ratio $\left(=1.76 \times 10^{11} \mathrm{~Hz} \cdot \mathrm{T}^{-1}\right.$, Landé factor $g=2)$. In the steady regime, the magnetization modulus is given by $\langle M\rangle \sim \int_{0}^{\infty} M d t$. Straightforward calculations lead to $\langle M\rangle=\langle\delta M\rangle \sim(\delta M / \delta t)\left[1 /\left(1+\left(\omega \tau_{\mathrm{dec}}\right)^{2}\right)\right]$, where $\omega=$ $\gamma\left(H_{x y}-H_{0 x y}\right)$ is the Larmor pulsation and $\delta M / \delta t$ the rate of formation of out-of-equilibrium magnetization. For $\delta M / \delta t$ assumed to be proportional to the particles current $(I / q)$ and energy barrier heights decreasing linearly with $\langle\delta M\rangle$, the mean dwell time reads

$$
\ln \left(\tau_{\text {mean }}\right)=\ln \tau_{0}+\frac{1}{k_{B} T}\left[\Delta E_{0}-\frac{a I}{1+\left(\omega \tau_{\mathrm{dec}}\right)^{2}}\right] .
$$

The only adjustable parameter $\tau_{\mathrm{dec}}$ for the magnetic field dependency in Eq. (3) can be directly deduced from a fit of experimental results, as shown in Fig. 3(b). The very good agreement supports a scenario of spin decoherence via the Hanle effect with a negligible anisotropy. $\tau_{\mathrm{dec}}$ equals $750 \pm$ $80 \mathrm{ps}$ and $660 \pm 70 \mathrm{ps}$, for $T=92 \mathrm{~K}$ and $T=98 \mathrm{~K}$, respectively. As $\tau_{\mathrm{dec}}>10^{4} \tau_{\mathrm{SF}}$, a magnetization reversal only driven by uncoupled carrier spins can be ruled out. In order to determine the origin of $\tau_{\mathrm{dec}}$, additional ferromagnetic resonance measurements were performed. As reported in Ref. [24], the inhomogeneous width characterizing the distribution of resonance frequencies of the coupled holeMn spins system was found to be $\Delta H_{\text {inh }}=70 \pm 10 \mathrm{Oe}$, for $T=100 \mathrm{~K}$. The corresponding dephasing time is $\tau_{\text {inh }}=1 /\left(\gamma \Delta H_{\text {inh }}\right) \approx 850 \pm 150$ ps. As $\tau_{\text {dec }}$ is close both to $\tau_{\text {inh }}$ and $\tau_{\text {relax }}$, the reduction of magnetization reversal rate observed in Fig. 3 can be attributed to the decoherence of the coupled out-of-equilibrium Mn-hole spins system. As expected, the observed nucleation process implies time scales much larger than the spin flip time of the uncoupled carrier spins.

In conclusion, the mechanism of current induced magnetization reversal in single semiconducting ferromagnets was shown to be very different from those proposed in the literature [18-22]. The nucleation of magnetization reversal is produced by a strong decrease of the magnetization and of the magnetic anisotropy. As a consequence, it would be particularly interesting to study to what extent the weakening of the interactions between local magnetic moments produced by carrier spins accumulation contributes to STT phenomena for metallic systems.

The authors wish to thank S. Rohart, H. Jaffrès, J.-M. Georges, C. Gourdon, and L. Thévenard for fruitful discussions and J. Miltat for his careful reading of the manuscript. This work was partly supported by the French projects DIM C'Nano IdF (Région Ile-de-France), 
ANR-MANGAS (No. 2010-BLANC-0424), and RTRA Triangle de la physique, Grant No. 2010033TSeMicMagII. This work was partly supported by the French RENATECH network.

*vincent.jeudy@u-psud.fr

[1] K. Pappert, S. Hümpfner, C. Gould, J. Wenisch, K. Brunner, G. Schmidt, and L. W. Molenkamp Nat. Phys. 3, 573 (2007).

[2] S. Mark, P. Dürrenfeld, K. Pappert, L. Ebel, K. Brunner, C. Gould, and L. W. Molenkamp, Phys. Rev. Lett. 106, 057204 (2011).

[3] S. S. P. Parkin, M. Hayahi, and L. Thomas, Science 320, 190 (2008).

[4] C. D. Stanciu, F. Hansteen, A. V. Kimel, A. Kirilyuk, A. Tsukamoto, A. Itoh, and Th. Rasing, Phys. Rev. Lett. 99, 047601 (2007).

[5] D. Chiba, M. Sawicki, Y. Nishitani, Y. Nakatani, F. Matsukura, and H. Ohno, Nature (London) 455, 515 (2008).

[6] T. Niazi, M. Cormier, D. Lucot, L. Largeau, V. Jeudy, J. Cibert, and A. Lemaître, Appl. Phys. Lett. 102, 122403 (2013).

[7] M. Tsoi, A. G. M. Jansen, J. Bass, W. C. Chiang, M. Seck, V. Tsoi, and P. Wyder, Phys. Rev. Lett. 80, 4281 (1998).

[8] E. B. Myers, D. C. Ralph, J. A. Katine, R. N. Louie, and R. A. Buhrman, Science 285, 867 (1999).

[9] Y. Ji, C. L. Chien, and M. D. Stiles, Phys. Rev. Lett. 90, 106601 (2003).

[10] E. B. Myers, F. J. Albert, J. C. Sankey, E. Bonet, R. A. Buhrman, and D. C. Ralph, Phys. Rev. Lett. 89, 196801 (2002).

[11] A. Fábián, C. Terrier, S. S. Guisan, X. Hoffer, M. Dubey, L. Gravier, J.-Ph. Ansermet, and J.-E. Wegrowe, Phys. Rev. Lett. 91, 257209 (2003).

[12] S. Urazhdin, N. O. Birge, W. P. Pratt, and J. Bass, Phys. Rev. Lett. 91, 146803 (2003).

[13] T. Y. Chen, Y. Ji, C. L. Chien, and M. D. Stiles, Phys. Rev. Lett. 93, 026601 (2004).

[14] D. Chiba, Y. Sato, T. Kita, F. Matsukura, and H. Ohno, Phys. Rev. Lett. 93, 216602 (2004).
[15] M. Elsen, O. Boulle, J.-M. George, H. Jaffrès, R. Mattana, V. Cros, A. Fert, A. Lemaître, R. Giraud, and G. Faini, Phys. Rev. B, 73, 035303 (2006).

[16] D. Ravelosona, S. Mangin, Y. Lemaho, J. A. Katine, B. D. Terris and E. E. Fullerton, Phys. Rev. Lett. 96, 186604 (2006).

[17] B. Özyilmaz and A. D. Kent, Appl. Phys. Lett. 88, 162506 (2006).

[18] L. Berger, Phys. Rev. B 549353 (1996).

[19] J. C. Slonczewski, J. Magn. Magn. Mater. 159, L1 (1996).

[20] M. D. Stiles and A. Zangwill, Phys. Rev. B 66, 014407 (2002).

[21] M. L. Polianski and P. W. Brouwer, Phys. Rev. Lett. 92, 026602 (2004).

[22] S. Zhang and Z. Li, Phys. Rev. Lett. 93, 127204 (2004).

[23] A. Lemaître, A. Miard, L. Travers, O. Maugin, L. Largeau, C. Gourdon, V. Jeudy, M. Tran, and J.-M. Georges, Appl. Phys. Lett. 93, 021123 (2008).

[24] See Supplemental Material at http://link.aps.org/ supplemental/10.1103/PhysRevLett.112.026601 for detailed information on the contribution of the Oersted field, Joule heating, ferromagnetic resonance measurements, spin lifetimes and diffusion lengths, and the model of magnetization decoherence by Hanle effect.

[25] J. Curiale, A. Lemaître, C. Ulysse, G. Faini, and V. Jeudy, Phys. Rev. Lett. 108, 076604 (2012).

[26] J. Curiale, A. Lemaître, T. Niazi, G. Faini, and V. Jeudy, J. Appl. Phys. 112, 103922 (2012); M. Cubukcu, H. J. von Bardeleben, Kh. Khazen, J. L. Cantin, O. Mauguin, L. Largeau, and A. Lemaître, Phys. Rev. B 81, 041202(R) (2010); M. Cubukcu, Ph.D. thesis, University Pierre and Marie Curie, 2010.

[27] T. Dietl, H. Ohno, and F. Matsukura, Phys. Rev. B 63, 195205 (2001).

[28] M. Wang, K. W. Edmonds, B. L. Gallagher, A. W. Rushforth, O. Makarovsky, A. Patane, R. P. Campion, C. T. Foxon, V. Novak., and T. Jungwirth, Phys. Rev. B 87, 121301 (2013).

[29] T. Valet and A. Fert, Phys. Rev. B 48, 7099 (1993).

[30] C. Rüster, PhD thesis, university Würzburg (2005).

[31] K. Shen and M. W. Wu, Phys. Rev. B 85075206 (2012). 